\title{
BANACH ALGEBRAS WITH UNCOMPLEMENTED RADICAL
}

\author{
GREGORY F. BACHELIS AND SADAHIRO SAEKI
}

\begin{abstract}
A condition is given on the invertible elements of a commutative Banach algebra $\mathbf{B}$ which implies that the radical of $\mathbf{B}$ is not complemented by any closed subalgebra. This condition is then applied to show that certain quotient algebras of group algebras have an uncomplemented radical.
\end{abstract}

I. Let $\mathbf{B}$ be a commutative complex Banach algebra with identity and let $\mathbf{S}$ be a closed ideal of $\mathbf{B}$. We say that $\mathbf{S}$ is algebraically complemented in $\mathbf{B}$ (by $\mathbf{C}$ ) if there exists a subalgebra $\mathbf{C}$ of $\mathbf{B}$ such that $\mathbf{B}=\mathbf{C} \oplus \mathbf{S}$. If a closed $\mathbf{C}$ can be found, we say that $\mathbf{S}$ is strongly complemented in $\mathbf{B}$. An invertible element $\mathbf{x} \in \mathbf{B}$ is said to be doubly power bounded (DPB) if the doubly infinite sequence $\left\{\left\|\mathbf{x}^{\mathbf{n}}\right\|: \mathbf{n} \in \mathbf{Z}\right\}$ is bounded. We denote the radical of $\mathbf{B}$ by $\mathbf{R}$. Our main result is as follows:

THEOREM 1. Suppose the DPB elements of $\mathbf{B}$ span a dense subspace and that $\mathbf{S}$ is a nonzero closed ideal of $\mathbf{B}$ contained in $\mathbf{R}$. Then $\mathbf{S}$ is not strongly complemented in $\mathbf{B}$.

Theorem 1 is applied to quotient algebras of group algebras to obtain a large class of Banach algebras with uncomplemented radical, as follows.

Let $\mathcal{G}$ be a nondiscrete locally compact abelian group, and let $\mathbf{A}(\mathcal{G})$ denote the Fourier algebra of $\mathcal{G}$ (see e.g. [15]). For $\mathcal{E}$ a compact subset of $\mathcal{G}$, let $\mathbf{I}(\mathcal{E})$ and $\mathbf{J}(\mathcal{E})$ denote, respectively, the largest and smallest closed ideals of $\mathbf{A}(\mathcal{G})$ with hull equal to $\mathcal{E}$. If $\mathbf{B}=\mathbf{A}(\mathcal{G}) / \mathbf{J}(\mathcal{E})$, then $\mathbf{R}=\mathbf{I}(\mathcal{E}) / \mathbf{J}(\mathcal{E})$. By definition, $\mathcal{E}$ is not of synthesis when $\mathbf{R} \neq(0)$. We have

THEOREM 2. If $\mathcal{E}$ is not of synthesis, then any nonzero closed ideal of $\mathbf{B}=$ $\mathbf{A}(\mathcal{G}) / \mathbf{J}(\mathcal{E})$ contained in $\mathbf{R}=\mathbf{I}(\mathcal{E}) / \mathbf{J}(\mathcal{E})$ is not strongly complemented in $\mathbf{B}$.

A compact subset $\mathcal{E}$ of $\mathcal{G}$ is said to be a Helson set if the restriction algebra, $\mathbf{A}(\mathcal{G}) \mathbf{I}(\mathcal{E})$ is all of $\mathbf{C}(\mathcal{E})$, the continuous functions on $\mathcal{E}$. By a theorem of Bade and Curtis [4, Theorem 4.1] we have

COROLlaRY 1. If $\mathcal{E}$ is a Helson set not of synthesis, then the radical of $\mathbf{B}=\mathbf{A}(\mathcal{G}) / \mathbf{J}(\mathcal{E})$ is not algebraically complemented in $\mathbf{B}$.

Helson sets not of synthesis exist in any $\mathcal{G}[\mathbf{1 9}]$. The above corollary was conjectured in $[\mathbf{1}]$.

In $\S I I$ we give the proofs of the above theorems. In $\S$ III vie make some remarks which include further results and connections between our results and the existing literature.

Received by the editors March 5, 1986. The results in this paper were presented to the Winter Meeting of the A.M.S., January 7, 1986. They are a portion of the results presented to the International Congress of Mathematicians, August 5, 1986.

1980 Mathematics Subject Classification. Primary 43A20, 46J35; Secondary 43A45, 43A46.

Second author supported in part by N.S.F. Grant DMS-8320479. 
II. We proceed to the proof of Theorem 1. Since the DPB elements are assumed to have dense span, it suffices to establish the following

LEMMA. Suppose that $\mathbf{S} \subset \mathbf{R}$ is a closed ideal of $\mathbf{B}$ which is strongly complemented by $\mathbf{C}$ and that $\mathbf{x}$ is a DPB element of $\mathbf{B}$. Then $\mathbf{x} \in \mathbf{C}$.

We use the following result of Gelfand.

THEOREM [7]. Let $\mathfrak{B}$ be a complex Banach algebra with identity $\mathbf{1}$, and let $\mathbf{x} \in \mathfrak{B}^{-1}$ be a DPB element with $\operatorname{sp}(\mathbf{x})=\{1\}$. Then $\mathbf{x}=\mathbf{1}$.

To prove the lemma, suppose that $\mathbf{x}=\mathbf{y}+\mathbf{z}$, where $\mathbf{y} \in \mathbf{C}$ and $\mathbf{z} \in \mathbf{S} \subset \mathbf{R}$. Let $\pi$ be the projection from $\mathbf{B}$ onto $\mathbf{C}$ with kernel $\mathbf{S}$. Then $\pi$ is a continuous homomorphism and $\pi(\mathbf{x})=\mathbf{y}$. Now any idempotent must be in $\mathbf{C}$. Thus $\mathbf{1} \in \mathbf{C}$, so $\pi\left(\mathbf{x}^{-1}\right)=\mathbf{y}^{-1}$ and $\mathbf{y}^{-1} \mathbf{x}=\mathbf{1}+\mathbf{y}^{-1} \mathbf{z}$. Since $\mathbf{y}^{-1} \mathbf{z} \in \mathbf{R}$, we have $\operatorname{sp}\left(\mathbf{y}^{-1} \mathbf{x}\right)=\{1\}$. Moreover, $\left\|\left(\mathbf{y}^{-1} \mathbf{x}\right)^{n}\right\| \leq\|\pi\|\left\|\mathbf{x}^{-n}\right\|\left\|\mathbf{x}^{n}\right\| \leq C$ for all $n \in \mathbf{Z}$ and some constant $C$. Thus Gelfand's Theorem implies $\mathbf{y}^{-1} \mathbf{x}=\mathbf{1}$, so $\mathbf{y}=\mathbf{x} \in \mathbf{C}$.

We now give the proof of Theorem 2. Let $\mathbf{B}=\mathbf{A}(\mathcal{G}) / \mathbf{J}(\mathcal{E})$. Choose and fix any $f \in \mathbf{A}(\mathcal{G})$ such that $f=1$ on some neighborhood of $\mathcal{E}$. Then $f+\mathbf{J}(\mathcal{E})$ is the identity of $\mathbf{B}$. Let $\Gamma$ denote the dual group of $G$. Then $\gamma \in \Gamma$ implies that $\gamma f+\mathbf{J}(\mathcal{E})$ is invertible in $\mathbf{B}$, and

$$
(\gamma f+\mathbf{J}(\mathcal{E}))^{n}=\gamma^{n} f+\mathbf{J}(\mathcal{E}) \quad \text { for all } \mathbf{n} \in \mathbf{Z} .
$$

Also,

$$
\left\|(\gamma f+\mathbf{J}(\mathcal{E}))^{n}\right\| \leq\left\|\gamma^{n} f\right\|_{A}=\|f\|_{A} \text { for all } n \in \mathbf{Z} .
$$

By Theorem 1 we only need show that the elements $\gamma f+\mathbf{J}(\mathcal{E}), \gamma \in \Gamma$, span a dense subspace of B. To this end, pick any $g \in \mathbf{A}(\mathcal{G})$. By Lemma 2 of [20], we can find sequences $\left(a_{k}\right) \subset \mathbb{C}$ and $\left(\gamma_{k}\right) \subset \Gamma$ such that $\sum_{k=1}^{\infty}\left|a_{k}\right|<\infty$ and $g=\sum_{k=1}^{\infty} a_{k} \gamma_{k}$ on some neighborhood of $\mathcal{E}$. Then

$$
g+\mathbf{J}(\mathcal{E})=\sum_{k=1}^{\infty} a_{k}\left(\gamma_{k} f+\mathbf{J}(\mathcal{E})\right)
$$

and the proof is complete.

If $\mathcal{E}$ is a Helson set, then $\mathbf{B} / \mathbf{R} \simeq \mathbf{A}(\mathcal{G}) / \mathbf{I}(\mathcal{E}) \simeq \mathbf{C}(\mathcal{E})$. If $\mathbf{R}$ were algebraically complemented in $\mathbf{B}$, then by Theorem 4.1 of $[4]$ it would be strongly complemented. By Theorem 2 this is impossible if $\mathcal{E}$ is not of synthesis. Thus Corollary 1 follows.

III. Remarks. We assume as before that $\mathbf{B}$ is a complex commutative Banach algebra with identity $\mathbf{1}$ and radical $\mathbf{R}$, that $\mathcal{G}$ is a nondiscrete LCA group, and that $\mathcal{E}$ is a compact subset of $\mathcal{G}$.

(1) If $\mathbf{e}$ is an idempotent in $\mathbf{B}$, then $\mathbf{1}-2 \mathbf{e}$ is a DPB element. Thus the DPB elements have dense span in $\mathbf{B}$ whenever the idempotents do. In [6] Feldman gave an example of a Banach algebra in which (i) the radical is not strongly complemented. In [3] Bade and Curtis showed that, for this example, (ii) the idempotents have dense span, and (iii) the radical is algebraically complemented. If one adjoins an identity to this example, then (i), (ii), and (iii) still hold. Thus "strongly complemented" cannot be replaced by "algebraically complemented" in Theorem 1 .

(2) In [14], Katznelson and Rudin proved, using results of $[\mathbf{3}]$, that if $\mathbf{A}(\mathcal{G}) / \mathbf{J}(\mathcal{E})$ is the closed span of its idempotents and $\mathcal{E}$ is not of synthesis, then the radical of 
$\mathbf{A}(\mathcal{G}) / \mathbf{J}(\mathcal{E})$ is not algebraically complemented. They state a more general result (Theorem 2.4) in which " $\mathbf{A}(\mathcal{G})$ " is replaced by any commutative regular Banach algebra without (closed nonmaximal) primary ideals. $\mathbf{A}(\mathcal{G}) / \mathbf{J}(\mathcal{E})$ is the closed span of its idempotents if $\mathcal{G}$ is totally disconnected or if $\mathcal{G}$ is the circle group and $\mathcal{E}$ has measure zero $[\mathbf{1 3}]$.

(3) In [16, Theorem 1] a condition is given for $\mathcal{E}$ to be a Helson set of synthesis which, for $\mathcal{E}$ totally disconnected, is readily seen to be equivalent to the statement that the idempotents in $\mathbf{A}(\mathcal{G}) / \mathbf{J}(\mathcal{E})$ are bounded. By [4, Theorem 2.4], if $\mathcal{E}$ is totally disconnected, then the idempotents of $\mathbf{A}(\mathcal{G}) / \mathbf{J}(\mathcal{E})$ are bounded iff $\mathcal{E}$ is a Helson set and $\mathbf{I}(\mathcal{E}) / \mathbf{J}(\mathcal{E})$ is algebraically complemented. Thus we obtain an alternate proof of Corollary 1 in the totally disconnected case.

(4) If $\mathcal{E}$ is a totally disconnected $F$-space (disjoint open $F_{\sigma}$ sets have disjoint closures [11]) then, as shown in [4], the idempotents in $\mathbf{A}(\mathcal{G}) / \mathbf{J}(\mathcal{E})$ are bounded, and hence $\mathcal{E}$ is a Helson set of synthesis. Thus we have a mildly different proof of the main result of $[\mathbf{1 8}]$. (In $[\mathbf{1 8}]$ it is stated that $\mathcal{E}$ must be extremally disconnected, but the proof only requires that $\mathcal{E}$ be a totally disconnected $F$-space.) The class of totally disconnected sets $\mathcal{E}$ for which the idempotents in $\mathbf{A}(\mathcal{G}) / \mathbf{J}(\mathcal{E})$ are bounded is strictly larger then the class of totally disconnected $F$-spaces $[\mathbf{9}]$.

(5) If $\mathcal{E}$ is a Helson set not of synthesis, then by Corollary 1 and [4, Theorem 4.2] $\mathbf{I}(\mathcal{E}) / \mathbf{J}(\mathcal{E})$ is not nilpotent. In fact, by the main result of $[\mathbf{8}]$, the sequence $\left\|\mathbf{r}^{n}\right\|^{1 / n}$ does not converge uniformly to zero on the unit ball of $\mathbf{I}(\mathcal{E}) / \mathbf{J}(\mathcal{E})$.

(6) It is easy to see that Theorem 2 and Corollary 1 remain valid when $J(\varepsilon)$ is replaced by any closed ideal $\mathbf{K}$ of $\mathbf{A}(\mathcal{G})$ such that $\mathbf{J}(\mathcal{E}) \subset \mathbf{K} \varsubsetneqq \mathbf{I}(\mathcal{E})$. When $\mathcal{E}$ is not of synthesis, there are lots of such closed ideals (see e.g. $[\mathbf{1 0}, \mathbf{1 7}, \mathbf{1 3}, \mathbf{2 1}]$ ).

(7) Gelfand's Theorem has a generalization due to Hille which is as follows [12, Theorem 4.10.1].

Let $\mathfrak{B}$ be a complex Banach algebra with identity 1 and let $\mathbf{x} \in \mathfrak{B}^{-1}$ with $\operatorname{sp}(\mathbf{x})=\{1\}$ be such that $\left\|\mathbf{x}^{ \pm n}\right\|=o\left(n^{N}\right)$ for some positive integer $N$. Then $(\mathbf{x}-\mathbf{1})^{N}=0$.

Using this result, the proof of Theorem 1 can be easily modified to obtain

THEOREM $1^{\prime}$. Suppose that the set of elements $\mathbf{x} \in \mathbf{B}^{-1}$ such that $\left\|\mathbf{x}^{ \pm n}\right\|=$ $o\left(\mathbf{n}^{N}\right)$ (where $N$ may depend on $\mathbf{x}$ ) spans a dense subspace of $\mathbf{B}$. If $\mathbf{S} \subset \mathbf{R}$ is a closed ideal of $\mathbf{B}$ which is strongly complemented, then $\mathbf{S}$ is the closure of its nilpotent elements.

For a quantitative generalization of Gelfand's Theorem, see [2].

(8) If $\mathfrak{X}$ is a compact space, and if $\mathbf{B} / \mathbf{R} \simeq \mathbf{C}(\mathfrak{X})$, then the existence of a nonclosed algebraic complement to $\mathbf{R}$ is equivalent to the existence of a discontinuous isomorphism of $\mathbf{C}(\mathfrak{X})$ into $\mathbf{B}$ (see [3]). Such isomorphisms are now known to exist (for certain B), assuming the Continuum Hypothesis [5].

\section{REFERENCES}

1. G. F. Bachelis, Some radical quotients in harmonic analysis, Radical Banach Algebras and Automatic Continuity (Long Beach, Calif., 1981), Lecture Notes in Math., vol. 975, SpringerVerlag, Berlin and New York, 1983, pp. 301-308.

2. G. F. Bachelisd and S. Saeki, Power bounded elements in a Banach algebra (in preparation).

3. W. G. Bade and P. C. Curtis, Jr., Homomorphisms of commutative Banach algebras, Amer. J. Math. 82 (1960), $589-608$. 
4. $\ldots$, The Wedderburn decomposition of commutative Banach algebras, Amer. J. Math. 82 (1960), 851-866.

5. H. G. Dales and J. Esterle, Discontinuous homomorphisms from $C(X)$, Bull. Amer. Math. Soc. 83 (1977), 257-258.

6. C. Feldman, The Wedderburn principal theorem in Banach algebras, Proc. Amer. Math. Soc. 2 (1951), 771-777.

7. I. Gelfand, Zur Theorie der Charaktere der Abelschen topologischen Gruppen, Rec. Math. (N.S.) 9 [51] (1941), 49-50.

8. E. A. Gorin and V. Y. Lin, On a condition on the radical of a Banach algebra ensuring strong decomposability, Mat. Zametki 2 (1967), 589-592.

9. A. Y. Helemskii, Example of a compact space which has the uniqueness property without being an F-space, Mat. Zametki 1 (1967), 735-740.

10. H. Helson, On the ideal structure of group algebras, Ark. Mat. 2 (1952), 83-86.

11. M. Henrikson, Some remarks on a paper of Aronszajn and Panitchpakdi, Pacific J. Math. 7 (1957), 1619-1621.

12. E. Hille and R. S. Phillips, Functional analysis and semigroups, Amer. Math. Soc. Colloq. Publ., vol. 31, Amer. Math. Soc., Providence, R.I., 1957.

13. J.-P. Kahane, Séries de Fourier absolument convergentes, Springer-Verlag, Berlin, 1970.

14. Y. Katznelson and W. Rudin, The Stone-Weierstrass property in Banach algebras, Pacific J. Math. 11 (1961), 253-265.

15. W. Rudin, Fourier analysis on groups, Interscience, New York, 1962.

16. S. Saeki, A characterization of SH-sets, Proc. Amer. Math. Soc. 30 (1971), 497-503.

17. __ An elementary proof of a theorem of Henry Helson, Tôhoku Math. J. 20 (1968), 244-247.

18. __ Extremally disconnected sets in groups, Proc. Amer. Math. Soc. 52 (1975), 317-318.

19. __ Helson sets which disobey spectral symthesis, Proc. Amer. Math. Soc. 47 (1975), 371-377.

20. __ Tensor products of Banach algebras and harmonic analysis, Tôhoku Math. J. 24 (1972), 281-299.

21. J. D. Stegeman, Extension of a theorem of Henry Helson, Proc. Internat. Congr. Math., Abstracts, Section 5 (1966), p. 28.

Department of Mathematics, Wayne State University, Detroit, Michigan 48202

Department of Mathematics, Kansas State University, Manhattan, Kansas 66506 